\title{
"Aha" effects in the generation of pictures
}

\author{
THEODORE W. WILLS, SAL A. SORACI, RICHARD A. CHECHILE, and HOLLY A. TAYLOR \\ Thifts University, Medford, Massachusetts
}

\begin{abstract}
An "aha" effect in memory was first reported by Auble, Franks, and Soraci (1979). They demonstrated that recall was greater for sentences that were initially incomprehensible but which were eventually comprehended, as compared with sentences that were understood from the outset. The present studies extend this "aha" effect to memory for pictorial stimuli. In Experiment 1, a recall advantage for pictures encoded by connecting the dots as compared with those encoded by tracing or visual scanning occurred only in the absence of foreknowledge of the picture (i.e., an "aha" effect). In Experiment 2, we replicated this finding and obtained evidence that conceptually based, verbal foreknowledge does not function in a similar manner as does pictorial foreknowledge in suppressing the "aha" recall advantage. These results place important constraints on previous research on generation effects for visual stimuli and attest to the cross-modal generalizability of the "aha" effect.
\end{abstract}

The generation effect is a robust cognitive phenomenon characterized by a memory advantage for items that are subject generated versus experimenter provided. The effect has been demonstrated with a wide range of experimental stimuli (Johns \& Swanson, 1988; Roenker, Wenger, Thompson, \& Watkins, 1978; Slamecka \& Graf, 1978; Soraci et al., 1994). To cite several examples, retention advantages due to generative processes have been observed with complex sentential stimuli (Graf, 1982), simple word fragments (Glisky \& Rabinowitz, 1985), homographs (McElroy, 1987; Soraci et al., 1999), and mathematical problems (McNamara \& Healy, 1995).

In terms of generalizability, a demonstration of the generation effect with pictorial stimuli would be of particular interest. In this paper, we examine generation effects obtained with visual stimuli and clarify the few extant findings in the literature. Of particular relevance are the results obtained in a series of experiments by Peynirciogglu (1989), in which both real and nonsense pictures that were constructed by following verbal instructions or connecting dots were recalled more frequently than those that had been traced or rated for artistic quality. Peynircioğlu interpreted the findings as evidence for the role of effortful processing in the generation effect for visual stimuli. However, these findings obtained with the connect-the-dots manipulation are amenable to an alternative interpretation.

This research was supported by the National Institute of Child Health and Human Development Grants KO4IIDOO921 and RO1 HD23682 awarded to Tufts University and the E. K. Shriver Center for Mental Retardation, Inc., respectively. This work was also supported in part by the Department of Mental Retardation of the Commonwealth of Massachusetts (Contract 100220023SC). We thank Stacy Arnovitz, Aaron Dalin, Jenn DePaul, Liz Monast, and Deborah Swibel for their assistance in collecting data. We would also like to express our appreciation to Peter Graf, Zehra Peynircioğlu, Jim Clark, and Keith Horton for their helpful comments and suggestions on earlier versions of this paper. Correspondence concerning this article should be addressed to T. Wills, Department of Psychology, Tufts University, Medford, MA 02155 (email: twills@emerald.tufts.edu).
Auble, Franks, and Soraci (1979) demonstrated what they termed an "aha" effect for sentential memory. They tested recall of sentences that were initially difficult to comprehend (e.g., "The man's back ached because the ends were too large"), but were subsequently made comprehensible by the presentation of a cue word "Barbell"). Recall in these cases was greater than when the sentence was comprehensible from the outset. The authors labeled this phenomenon the "aha" effect and speculated that the transition from a state of noncomprehension to a state of comprehension was mediating the recall advantage.

Similarly to an ambiguous sentence, a pattern of dots is initially incomprehensible. When the dots are being connected, there is some critical point at which the figure becomes identifiable. It is possible that Peynircioğlu's (1989) subjects were exhibiting superior recall for connecting the dots, as compared with tracing, simply because of the transition from noncomprehension to comprehension of a global visual form, rather than the act of connecting the dots per se. If true, this would suggest that the operation of the "aha" effect could have played a critical and unexamined role in Peynircioğlu's study.

Experiment 1 was designed to explore the possible role of "aha" in mediating the recall advantage reported by Peynircioğlu (1989). A novel manipulation involving a preview of the to-be-completed figure was used to determine whether generation, "aha," or a combination of the two might be operating in the connect-the-dot paradigm. Furthermore, we sought to clarify the role of semantic processing in memory of pictures by including nonsense figures that, unlike the nonsense figures that Peynircioğlu used, were not conducive to propositional processing.

\section{EXPERIMENT 1}

\footnotetext{
Method

Subjects. A total of 48 subjects took part in the study. The subjects were Tufts University undergraduates, who received partial class credit.
} 

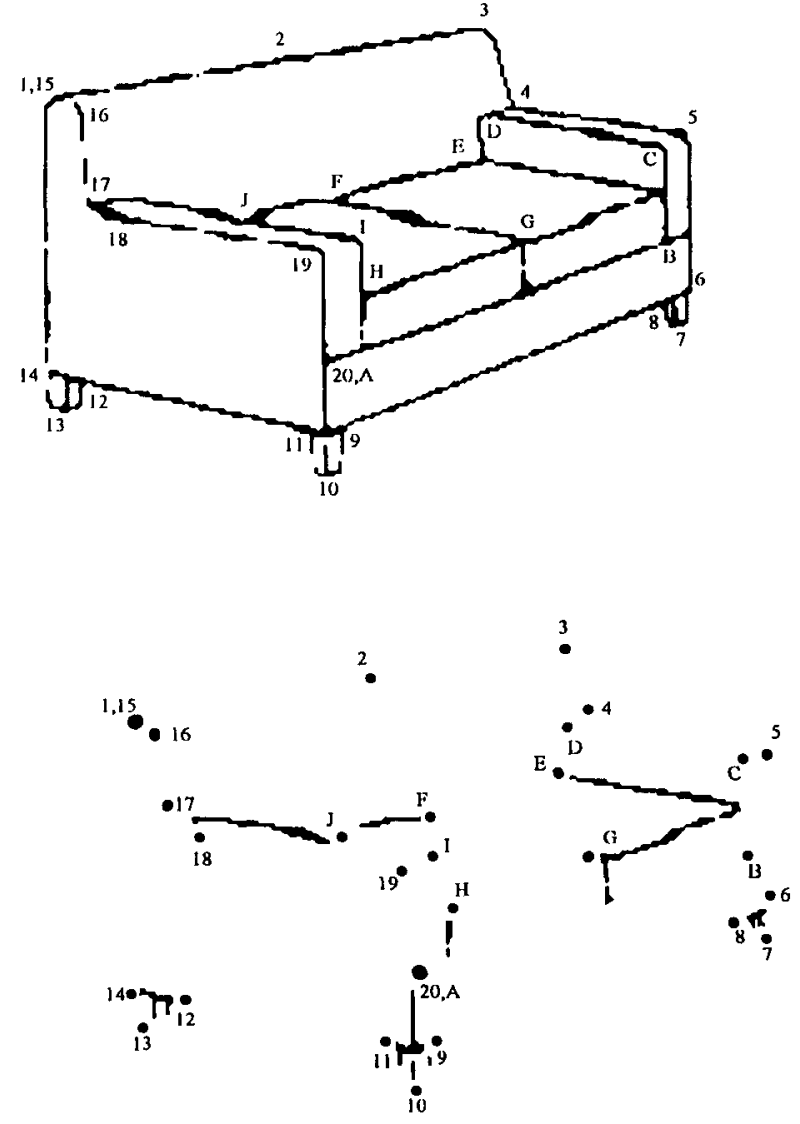
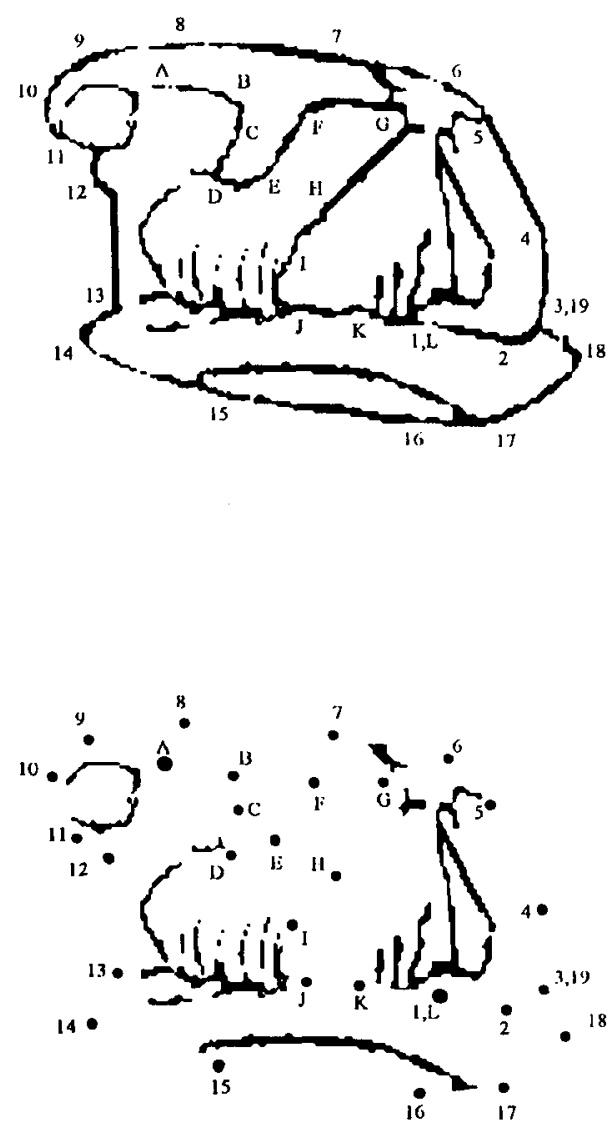

Figure 1. Example of connect the dots and trace/scan versions of one real and one nonsense figure.

Materials. The learning set consisted of 40 line drawings of objects. Half of the pictures, termed real, were line drawings of common, everyday objects (e.g., a couch, a swing, or a dress). The remaining 20 pictures, the nonsense pictures, depicted objects that do not exist. Unlike the nonsense figures used by Peynircioğlu (1989), which were amenable to propositional processing, the nonsense pictures were complicated line drawings, similar in style and level of detail to the real pictures, but not corresponding to existing objects. In this way, the nonsense pictures were similar to the real pictures on as many dimensions as possible, without being conducive to propositional or linguistic encoding.

Two versions of each picture were constructed. The first, the dot version, was designed to be completed via a connect-the-dots task. These pictures were composed of several dots, each labeled with a number or a letter. The other version was simply the original line drawing plus the numbers and letters that were present in the dot version. In pilot testing, none of the volunteers was able to identify the final form of the connect-the-dots pictures from the initial pattern of dots (see Figure 1).

For each picture in the learning set, a four-alternative recognition array was constructed. One of the four alternatives, the target, was the original picture. The other three were constructed by manipulating the original picture in one or more of the following ways: (1) reversing the orientation (i.e., presenting the mirror image); (2) adding a feature consistent with the object, but not present in the original; or (3) deleting a feature not critical to the identity of the object that was present in the original. A single foil could display one, two, or all three of these manipulations, for a total of seven possible foil types. Each array was presented on a standard $8.5 \times$ 11 in. piece of paper, with one picture in each quadrant (see Figure 2). Across the set of 40 arrays, each type of foil was present with comparable frequency and with comparable frequency in each array position. Likewise, the location of the target was counterbalanced throughout the set.

\section{Procedure}

The experimental design was a 3 (learning task) $\times 2$ (presentation order) $\times 2$ (object class) factorial arrangement, with learning task between subjects and the other factors within subjects. The subjects were given a series of 40 pictures to memorize, followed by a free recall task, and then by a recognition task. Task was a between-subjects factor, with 16 subjects assigned to each of the three levels, dot, trace, and scan. The subjects in the dot condition received the pictures as patterns of numbered and lettered dots. They then connected the numbered dots in sequence, followed by the lettered dots in alphabetical order to form the complete target picture. The subjects in the trace condition received the picture already completed, and their task was to use the numbers and letters as a guide for tracing the picture. The subjects in the scan condition also received the complete picture, and their task was to simply say the entire sequences of numbers and letters aloud while scanning them visually. The scan task was included for two reasons. First, it would serve the same function as Peynircioglu's (1989) artistic judgment task, as an encoding condition with no drawing element, allowing us to examine the contribution of drawing on subsequent memory. Second, it would improve the artistic rating task by forcing the sub- 

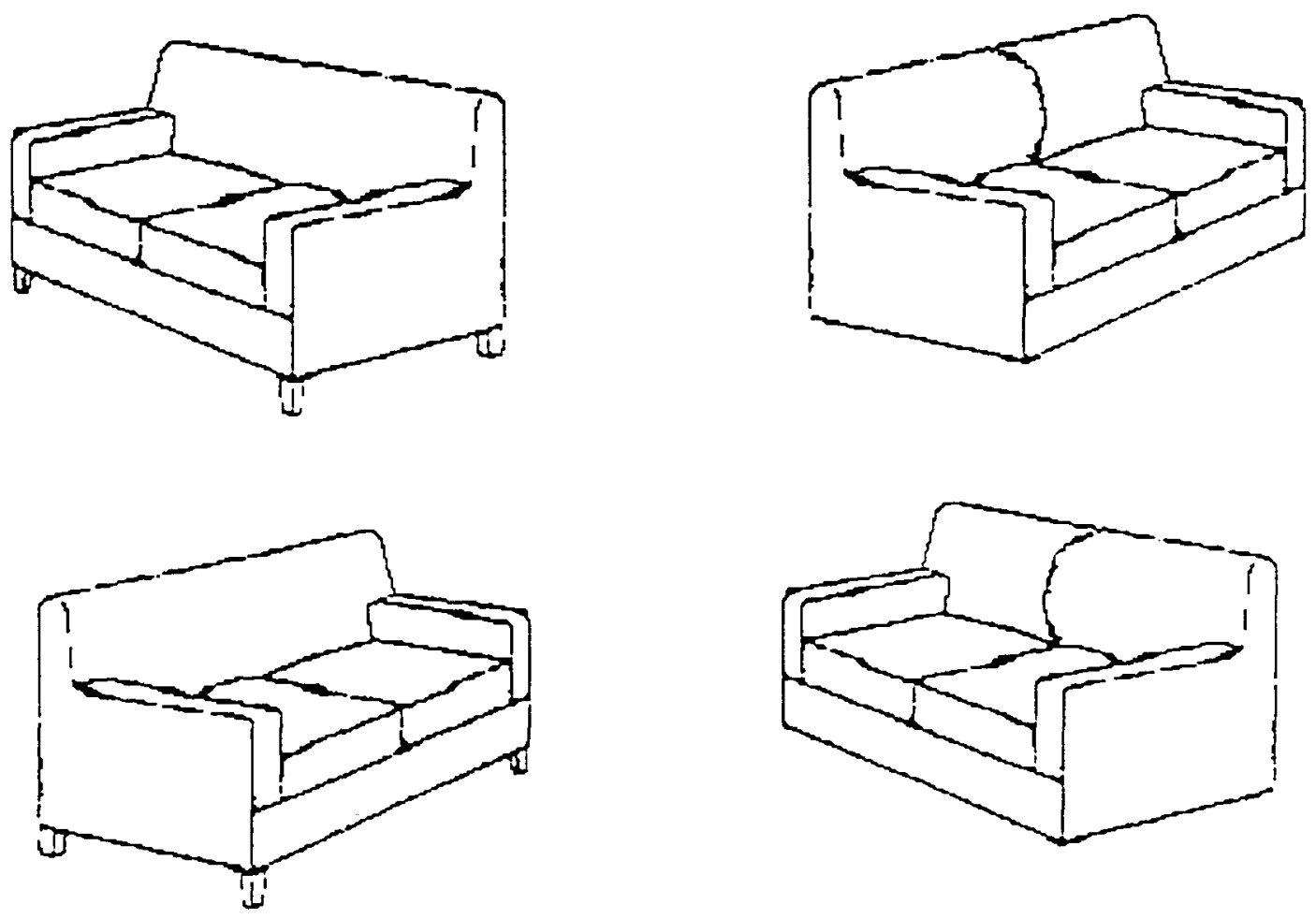

Figure 2. Example of a four-choice recognition array.

jects to focus on the local features of the picture, and to do so in the same sequence as the subjects in the trace and dots conditions. If Peynircioglu's finding of an advantage of trace over artistic judgment was driven by some contribution of drawing (as she surmised), we should find the same pattern. If, however, that advantage was an artifact of the judgment task (e.g., attention is drawn to the picture's global composition), it should not be replicated here. To control overall time of acquisition, the subjects in all three conditions were given $30 \mathrm{sec}$ to complete their task for each figure. Pilot data suggested, however, that the scan and trace subjects would, on the average, require less time to perform their task, so we were concerned that the subjects in these conditions might benefit from extra exposure in the absence of a task relative to those in the dots condition. To control for this potential over-learning, the subjects were given only $4 \mathrm{sec}$ to examine the picture after the completion of the task, at which time it was removed. If the 30-sec allotment had not yet expired, the remainder was allowed to elapse before the next figure was presented. In this way, overall time of the acquisition phase was equated while differential over-learning of individual figures was minimized.

To examine the role of the "aha" effect in the task, the presentation of the figures was further manipulated by a within-subjects factor with two levels, preview and postview. Each trial consisted of a presentation of a complete picture and the performance of a subject's task. In before trials, the complete picture was presented for $5 \mathrm{sec}$ and then removed. This first presentation was immediately followed by the task presentation, as described previously. In after trials, the task was performed first, and the 5-sec presentation of the complete picture followed.

Finally, in order to better understand the role of semantic processing in pictorial memory effects, the stimuli belonged to either of two classes, real and nonsense. Overall, the sequence of pictures was pseudo-randomized so that each block of four trials consisted of one each of the four combinations of real/nonsense and preview/ postview.

Acquisition was immediately followed by a free recall task, in which the subjects were given blank sheets of graph paper and asked to draw as many of the pictures as they could remember. They were asked to be as accurate and detailed as they could be. The subjects were given $5 \mathrm{~min}$ for this phase, though most required less time, and none requested more.

Immediately following the free recall phase, the subjects completed a recognition task. For each of the 40 pictures, they were given an array of 4 choices and were asked to identify which version of the picture matched the one seen during acquisition. The subjects were given $8 \mathrm{~min}$ to complete the recognition phase, and all subjects completed it in the allotted time.

\section{Results and Discussion}

Free recall. The subjects' drawings from the free recall phase of the experiment were scored by three raters. Efforts to rate the figures for accuracy were unsuccessful, because reproductions were almost universally poor. Because the subjects were not unusually talented at drawing, most of the pictures were distortions of the originals, and thus ratings of accurate reproduction would not necessarily reflect accurate recall. Therefore, a strict scoring criterion was adopted, under which a figure was judged to be successfully recalled if all three raters agreed on which figure the subject had attempted to reproduce. 


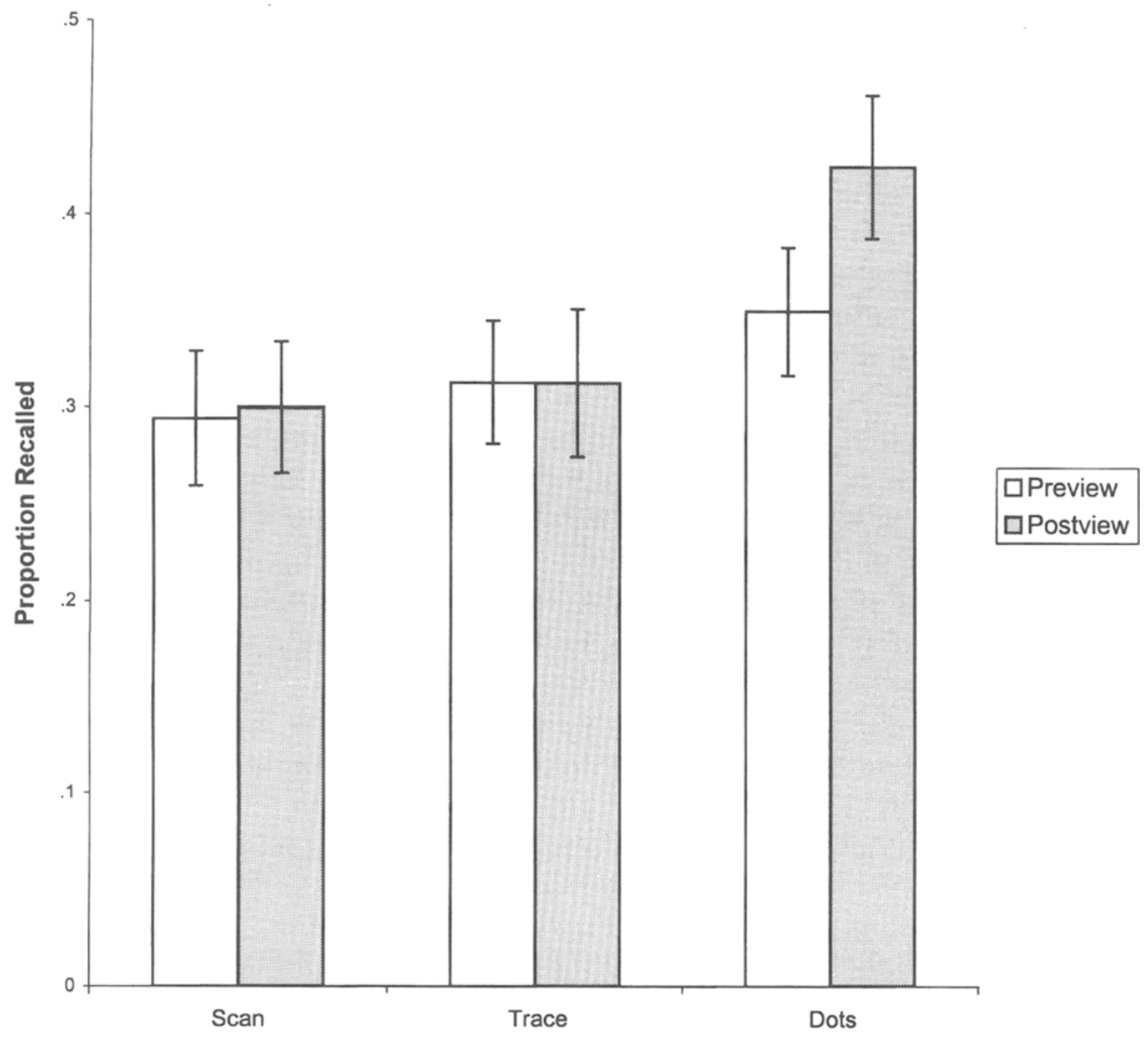

Figure 3. Proportion of real pictures recalled in Experiment 1, with error bars showing standard error of the mean.

That is, it was not necessary for the subject to render a version of the original picture that was accurate in detail or orientation, only that the intended picture was identifiable from the pool of figures presented during acquisition. The figures were also scored under a more lenient criterion, wherein agreement of only two of the raters was required. This was done simply to ensure that results were not an artifact of the strict scoring system. However, pictures were easily identified, with only 25 of the 395 figures that satisfied the lenient scoring criteria failing to meet the strict criteria (roughly $7 \%$ ). Not surprisingly, therefore, analyses of strict and lenient scorings were similar in substance. For this reason, and because it is the more stringent criterion, only the strict scoring is reported here. Alpha was set at $p=.05$ for all tests.

Not surprisingly, the main effect for picture type was highly significant, with real pictures recalled much more frequently $(35.0 \%)$ than nonsense figures $[6.5 \% ; F(1,45)=$
235.75, $\left.M S_{\mathrm{e}}=1.59, p<.001\right]$. In fact, nonsense figures were recalled so poorly that further interpretation would be suspect at best, so they were excluded from the remaining recall analyses.

Among the remaining main effects and interactions, only the main effect of task reached significance. Recall (for real pictures only) was greater in the dots condition $(38.8 \%)$ than in both the trace $(29.7 \%)$ and scan $(31.3 \%)$ conditions $\left[F(2,45)=3.65, M S_{\mathrm{e}}=1.30, p<.05\right]$. Although this is consistent with Peynircioğlu's (1989) findings, planned comparisons support a different interpretation. The dot advantage existed only in the case of real figures in the postview condition, where the subjects recalled more pictures $(44.4 \%)$ than either trace $(31.9 \%)$ or $\operatorname{scan}[30.0 \% ; t(30)=2.06, p<.05$ and $t(30)=2.40, p<$ .03 , respectively]. In other words, if the subject had foreknowledge of the figure, the recall advantage vanished (see Figure 3 ). The act of connecting the dots per se did not lead 


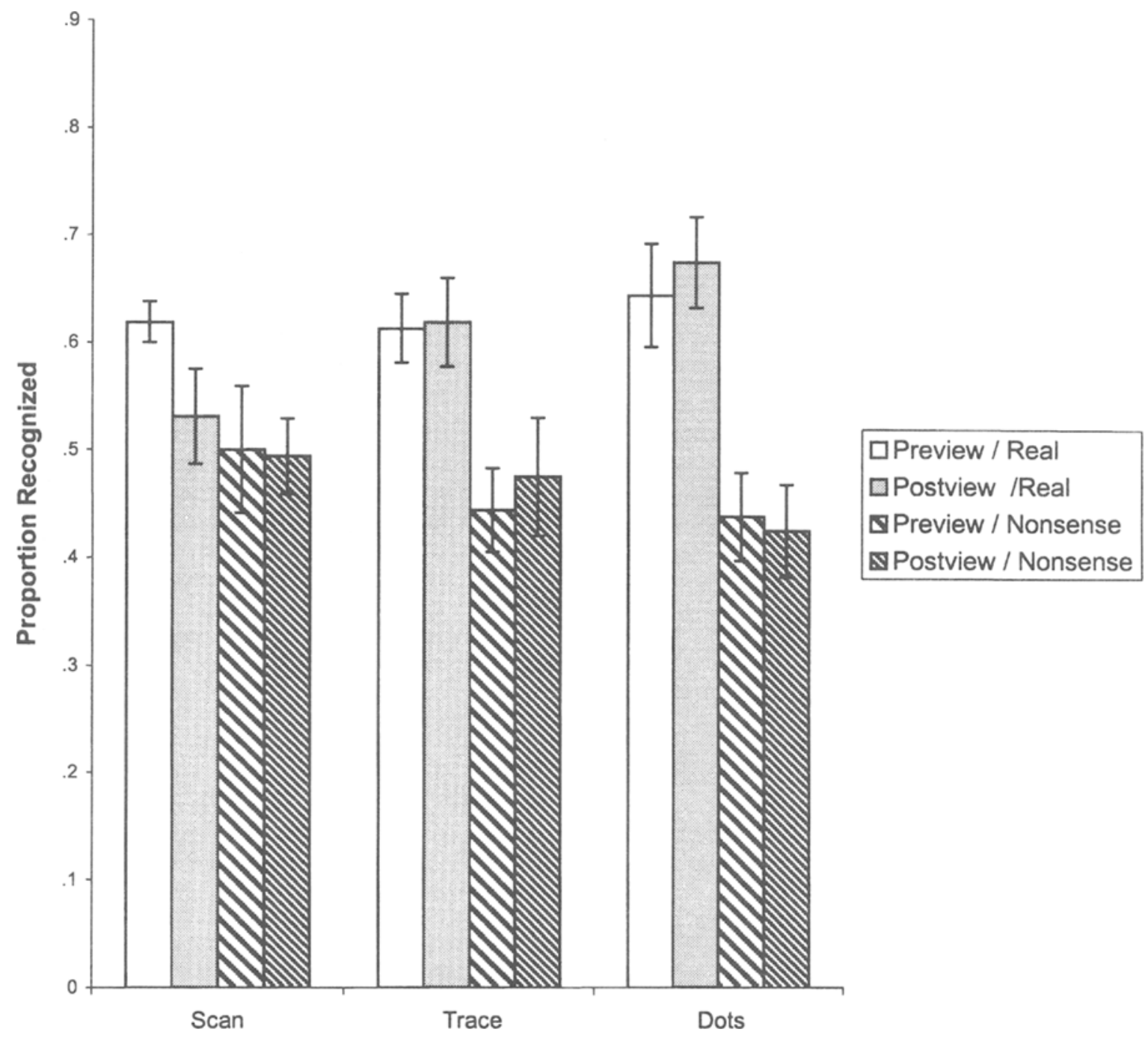

Figure 4. Proportion of pictures correctly recognized in Experiment 1, in four-choice arrays, with error bars showing standard error of the mean. Solid bars indicate real figures; hatched bars indicate nonsense figures.

to superior recall; it was also necessary that the subjects "decipher" an ambiguous form, a finding consistent with an explanation based on the "aha" effect.

Recognition. Recognition scores were obtained via subjects' performances on the multiple-choice picture arrays. These data were subjected to the same three-way factorial analysis of variance (ANOVA) as were the recall data (see Figure 4). Unlike for recall, no task-based advantages were observed. Again, the main effect for picture type reached significance $[F(1,45)=39.22$, $\left.M S_{\mathrm{e}}=2.91, p<.01\right]$, with real pictures recognized more frequently $(61.7 \%)$ than nonsense pictures $(46.3 \%)$. This recognition difference, however, could be a product of different levels in similarity in the foil sets of the two classes of figure. Although the absolute changes were comparable (mirror reversals, addition and subtraction of single features), we cannot be certain that these physical changes led to comparable changes in perceived similarity. No other main effect or interaction reached significance. It is worth noting that given the literature regarding congruence of motor activity at acquisition and test (e.g., Engelkamp, Zimmer, Mohr, \& Sellen, 1994), the trace condition might have been expected to produce higher performance than the scan condition.

\section{EXPERIMENT 2}

In Experiment 1, previews of to-be-completed real figures suppressed subsequent memory. In this case, foreknowledge of the target was "total," in the sense that the exact picture was provided at the beginning of the trial. Suppression of aha-type processes could have been driven by semantic knowledge, pictorial information, or both. It was therefore possible to clarify this ambiguity in Experiment 2 by providing a verbal label as a preview instead of a picture. In this way, pictorial foreknowledge 
could be withheld while semantic information was still provided.

\section{Method}

Subjects. Sixty-four students were subjects. Six were students enrolled in a summer session introductory psychology course and participated as volunteers. The remainder were undergraduate students enrolled in introductory psychology or psychological statistics and received partial course credit for participating. Data for 2 subjects in the word condition were later discarded because of experimenter error, leaving a total of 32 picture and 30 word subjects.

Materials. The 20 real figures from Experiment 1 were supplemented with 8 new real figures. As in Experiment 1, a connect-thedots version, a trace version, and a four-choice recognition array were generated for each figure. Study lists were generated in such a way that for each subject, half of the 28 figures were in the dot condition and half were in the trace condition. Furthermore, half of the figures were in the preview condition and half in the postview condition, so that there were seven figures in each of the four factorial combinations of task and sequence. Four lists were constructed in such a way that each figure was rotated through each of these four conditions with equal frequency across subjects. Finally, for each picture, a one-word label was generated and printed on an $8.5 \times 11$ in. piece of paper. In the word condition, this label replaced the secondary picture presentation.

The appropriateness of the picture-label matches were rated by volunteers who were naive to the purposes of the ratings and of the connected experiments. The 28 real figures, which were intended to be canonical, typical depictions of real-world objects, were combined with 14 pictures that were intended to be unusual, non-canonical depictions of other real-world objects. These pictures were arrayed, six to a page, together with the single word that was thought to best represent the picture (e.g., "padlock" or "couch"). The full set of pictures and labels, comprising 42 objects, were then given to 17 volunteers. These volunteers (a convenient sample including graduate and undergraduate students at Tufts University) were instructed to read the label and to rate the canonicality of the associated picture on a 4-point scale (1, very typical; 2 , somewhat typical; 3, somewhat atypical; 4, very atypical).

The ratings were consistent with expectations; pictures designed to be typical were consistently rated as such, and those intended to be non-canonical were rated as atypical. Canonical pictures received a mean rating of 1.17 , with a range of 1.00 to 1.41 . In contrast, non-canonical pictures received a mean rating of 2.99 , with a range of 2.06 to 3.65 . On the basis of these ratings, the labels were viewed as appropriate and were retained.

\section{Procedure}

The experimental procedure was identical to that of Experiment 1 , except for the following modifications. The nonsense pictures were eliminated, and only real pictures were used in Experiment 2. Furthermore, since there were no differences between the scan and trace conditions in Experiment 1, the scan condition was eliminated. Whereas dots and trace conditions were a between-subjects factor in Experiment 1, they were presented as a within-subjects factor in Experiment 2, with the subjects performing the dots task on half of the figures and the trace task on the other half. The final difference was a between-subjects manipulation of the mode of the secondary presentation. Half of the subjects served in the word condition, wherein the secondary presentation took the form of a verbal label, rather than of a redundant presentation of the target picture. The remaining subjects, in the picture condition, received the redundant picture presentation that was given to all subjects in Experiment 1 .

\section{Results and Discussion}

Free recall. Recall figures were subjected to the same strict and lenient scoring processes used in Experiment 1 , and as described previously. Of the 733 figures that satisfied the criteria for lenient recall, only 21 (about $3 \%$ ) failed to meet the more stringent requirements of strict recall. It is not surprising, therefore, that the results obtained from the strict ratings were similar in substance to those of the lenient ratings. Since there were no meaningful differences, and since it represents the more stringent criterion, the strict scoring analysis is reported here.

Recall measures were subjected to a 2 (task) $\times 2$ (presentation order $) \times 2$ (mode) ANOVA. Task and presentation order were analyzed as within-subjects variables, whereas mode was treated as a between-subjects variable (see Figure 5). The analysis revealed main effects of both task and presentation order. As in Experiment 1, the subjects demonstrated better recall for figures that had been produced by connecting the dots $(48.3 \%)$ than for those that had been traced $\left[34.1 \% ; F(1,60)=36.41, M S_{\mathrm{e}}=1.67\right.$, $p<.001]$. Furthermore, the subjects were more likely to recall figures when the secondary presentation came as a postview $(43.4 \%)$ rather than as a preview [39.0\%; $\left.F(1,60)=4.95, M S_{\mathrm{e}}=1.21, p<.03\right]$. There was, additionally, a presentation order $\times$ task interaction $[F(1,60)=$ $\left.7.12, M S_{\mathrm{e}}=1.78, p<.01\right]$ with the advantage for connecting the dots depending on whether there was a preview or postview. There was a suggestion that the mode of the pre- and postviews was important; the three-way interaction approached significance $[F(1,60)=3.25$, $\left.M S_{\mathrm{e}}=1.78, p<.08\right]$. Essentially, the suppression of "aha" was greater when the preview was a picture than when it was a lable.

Post hoc analyses supported and clarified the preceding results. Focusing first on the picture condition only, we found that the pattern observed in Experiment 1 was replicated. Connecting the dots led to greater recall than tracing, but only if there was no preview of the final picture. For connecting the dots with a postview, recall was greater $(54.3 \%)$ than for tracing with a preview [35.9\%; $t(29)=3.87, p<.001]$ and tracing with a postview $[28.4 \% ; t(29)=6.49, p<.001]$. This advantage also held relative to connecting the dots with a preview [39.8\%; $t(29)=4.03, p<.001]$. Finally, the apparent difference between tracing with a preview and tracing with a postview was not reliable $[t(29)=1.50, p>.10]$.

The pattern was different in the word condition. Here, connecting the dots with a postview still provided improved memory $(53.1 \%)$ over tracing with a preview $(34.4 \%)$, or a postview $[37.6 \% ; t(31)=5.19, p<.001$ and $t(31)=3.66, p<.01$, respectively]. However, unlike the results obtained with a pictorial preview, the connect-thedots advantage was not suppressed by a verbal preview $(45.6 \%)$ relative to tracing with a verbal preview $[t(31)=$ $2.22, p<.05]$ and approached significance relative to tracing with a verbal postview $[t(31)=1.78, p<.10]$. 


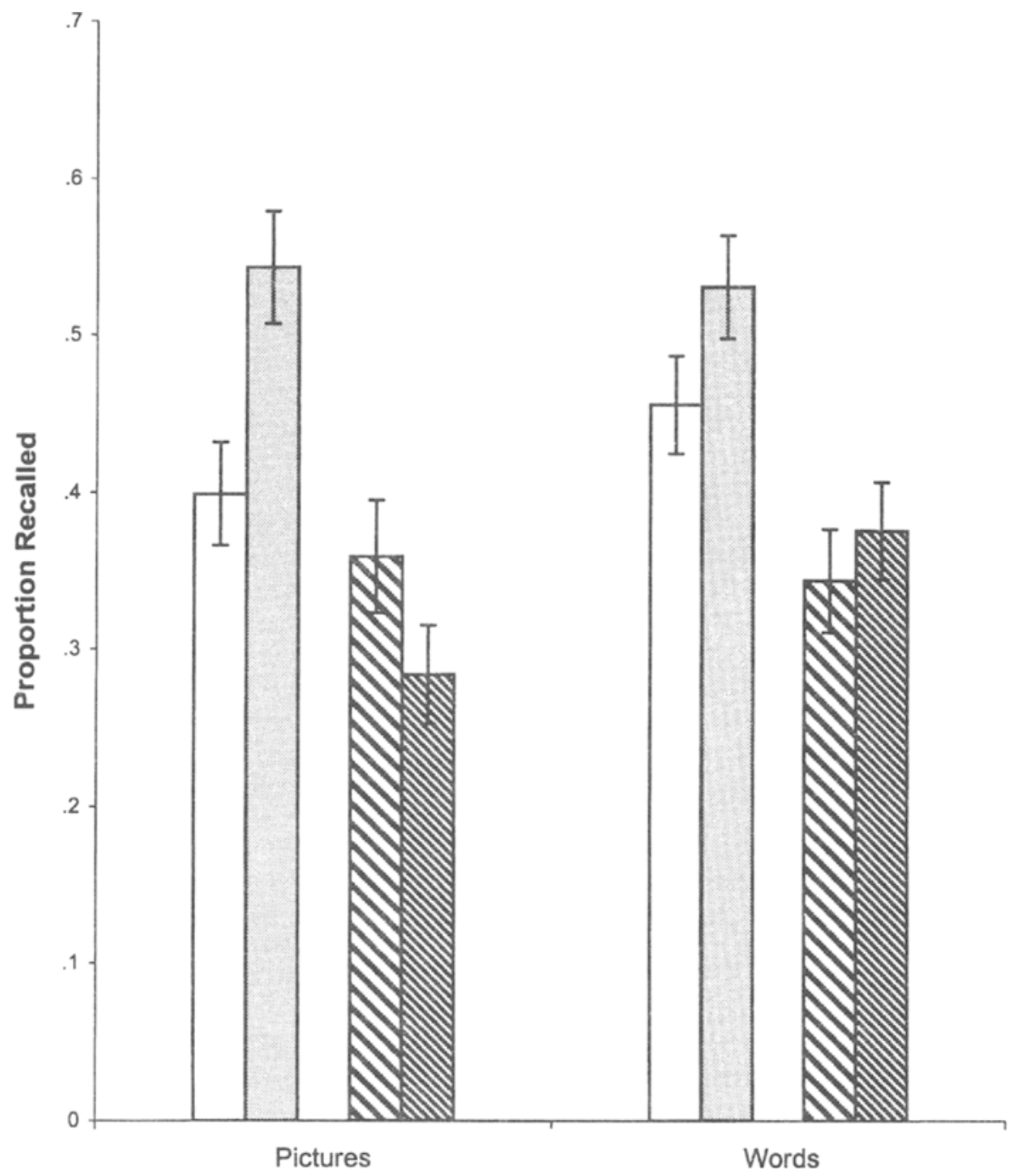

口Preview / Dots
$\square$ Postview / Dots
QPreview / Trace
\$Postview / Trace

Figure 5. Proportion of pictures recalled in Experiment 2, with error bars showing standard error of the mean. Solid bars indicate recall of figures that were formed by connecting the dots; hatched bars correspond to figures that were traced.

Furthermore, there was no advantage for connecting the dots with a verbal postview over connecting the dots with a verbal preview $[t(31)=1.10, p>.20]$.

Of secondary interest, there was no effect of mode, with subjects in the word and picture conditions recalling the same number of figures overall $[F(1,60)=1.53$, $\left.M S_{\mathrm{e}}=1.83, p>.20\right]$. Furthermore, mode $\times$ sequence and mode $\times$ task interactions did not reach significance.

Recognition. The subjects' performance on the fourchoice recognition arrays was quite different from what was observed in Experiment 1 (see Figure 6). Of greatest significance was the finding that there was a main effect of task, with figures obtained by connecting the dots recognized more accurately $(64.0 \%)$ than those obtained by tracing $\left[54.7 \% ; F(1,60)=9.87, M S_{\mathrm{e}}=2.69, p<.005\right]$. This could be interpreted as a "pure" generation effect for pictorial stimuli, driven by the connect-the-dots task. In Experiment 1, where task was a between-subjects variable, no such pattern was seen. Within-subjects designs are frequently more sensitive with respect to the generation effect (McDaniel, Waddill, \& Einstein, 1988), and it may be that this is why Experiment 2 was able to detect a difference that did not appear in Experiment 1.

Furthermore, there was a significant main effect of mode, with the subjects that received pictorial pre- and postviews correctly recognizing more figures $(64.0 \%)$ than those who received verbal labels $[54.9 \% ; F(1,60)=$ $\left.8.61, M S_{\mathrm{e}}=2.96, p<.05\right]$. This advantage is consistent with a transfer-appropriate processing perspective (Morris, Bransford, \& Franks, 1977). It is reasonable to assume that the subjects in the word and picture conditions approached their tasks in different ways. Specifically, exposure to the label may have made the semantic component of the picture more salient, at least in comparison with redundant exposure to the picture. From this view, acquisition and recognition would be highly congruent 


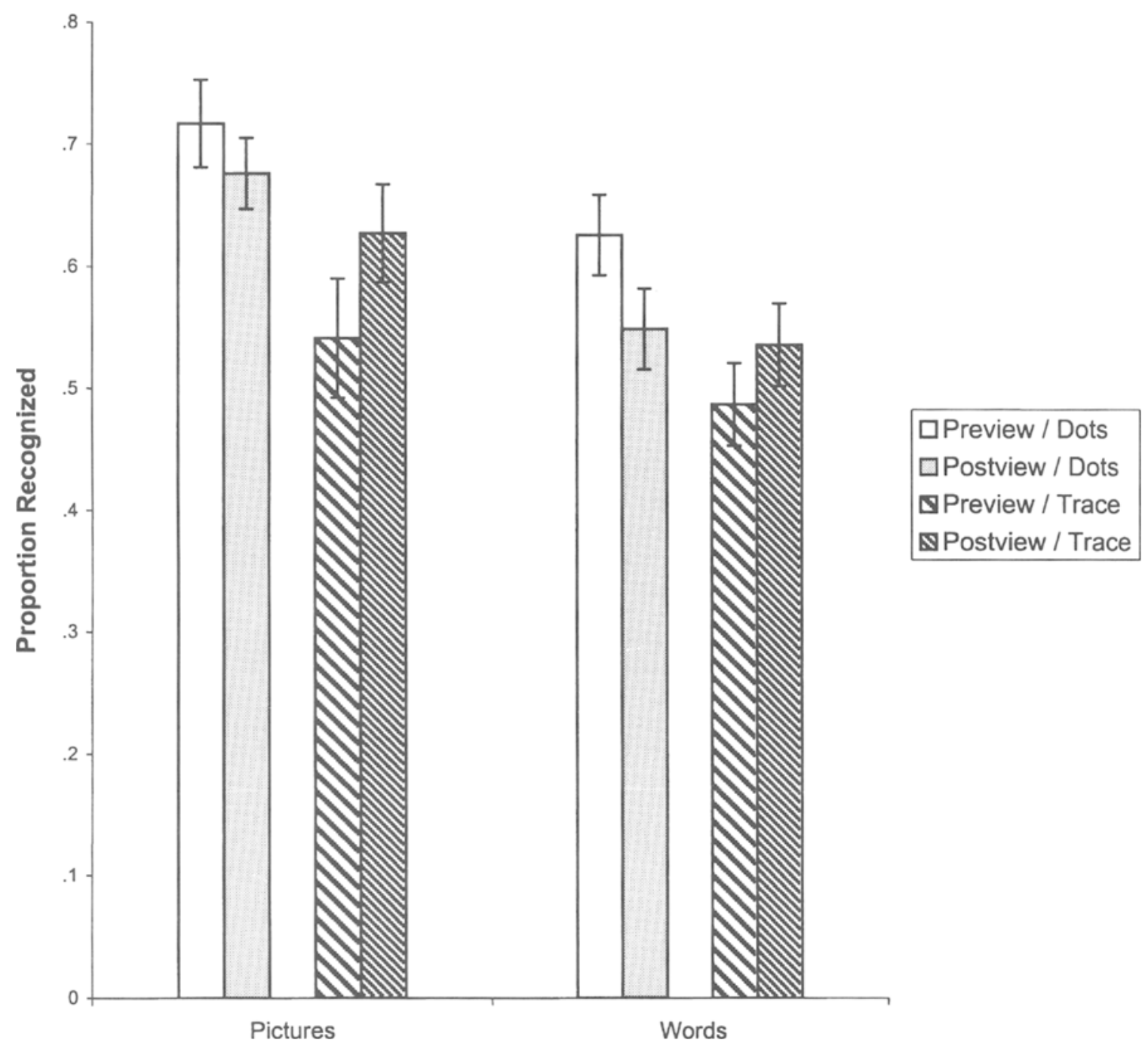

Figure 6. Proportion of pictures correctly recognized in Experiment 2, in four-choice arrays, with error bars showing standard error of the mean. Solid bars indicate recognition of figures that were formed by connecting the dots; hatched bars correspond to figures that were traced.

learning and test phases for the picture subjects, and less so for the word subjects. Finally, it is important to note that this advantage could be due to simple exposure. In the picture condition, the subjects had the benefit of a second presentation of the picture. This account is somewhat problematic, however, since the 5-sec view was quite brief relative to the difference in exposure between the trace and dots tasks. The effect of task, however, was directly opposite to that predicted by simple exposure. Trace subjects (who viewed the complete picture for the duration of the task) performed less well than the dots subjects, who had, at most, $4 \mathrm{sec}$ to view the completed picture during the performance of the task.

There was no main effect of presentation order, with pre- and postviews leading to comparable recognition $\left[F(1,60)=.06, M S_{\mathrm{e}}=0.80, p>.80\right]$. However, this null finding was mitigated by a significant sequence $\times$ task interaction $\left[F(1,60)=6.99, M S_{\mathrm{e}}=1.73, p<.02\right]$. For figures that were traced, there was greater recognition for pictures that had postviews, whereas previews led to greater recognition of figures that appeared in the connectthe-dots condition.

None of the remaining interactions approached significance.

\section{Summary}

In Experiment 2, we replicated and extended the findings of Experiment 1 . The recall measures provided two key findings. The first was a replication of Experiment 1 with respect to the picture condition. Despite the change of task from a between- to a within-subjects manipulation, and despite the elimination of nonsense figures, the same pattern of results emerged. The recall advantage for connecting the dots was suppressed when a picture 
preview was provided. Again, despite optimal conditions for detecting a task-based generation effect, the observed retention advantage was better subsumed under the "aha" effect. The second key finding was that verbal foreknowledge of a to-be-completed picture does not function, in this paradigm, in the same way as "visual" foreknowledge. Providing a verbal label preview did not eliminate the connect-the-dots advantage.

\section{GENERAL DISCUSSION}

In the present experiments, we extended the "aha" effect demonstrated by Auble et al. (1979) with sentential stimuli to memory for pictorial stimuli and delineated a critical variable unexamined in Peynircioğlu's (1989) original study. What mechanisms might mediate an "aha" effect? As an individual attempts to encode an ambiguous stimulus, there is a generation of alternative response candidates (see Bruner \& Potter, 1964). Soraci et al. (1994) demonstrated this with a paradigm requiring generation of responses that were incongruous with respect to an encoding rule. They found with both protocol and recall indices that subjects entertained intermediate "incorrect" responses that were activated and rejected during the attempt to arrive at a correct response. These additional items were predicted to function as effective retrieval cues during recall. In a series of five studies, Soraci et al. (1994) provided strong evidence in support of this multiple-cue hypothesis for incongruous item generation effects. Recently, Smith and Healy (1998) reported enhanced recall for delayed solutions in certain problem-solving conditions and claimed that, consistent with Soraci et al.'s (1994) multiple-cue hypothesis, the additional encoding time facilitated cue activation. In summary, converging evidence from several studies supports the hypothesis that effort toward comprehension, especially in encoding contexts in which the solution is uncertain, induces the activation of alternative cues that can later facilitate recall.

Another important finding was that a task-based generation effect did not occur in recall, but did emerge in the recognition phase of Experiment 2. Likewise, an "aha" effect was observed in recall in both studies, but not in recognition. This differential pattern across retention tests is actually quite interpretable. According to a transferappropriate processing perspective (Morris et al., 1977), free recall is similar to the generative processes occurring at acquisition. Also, in the present studies, free recall measures were particularly sensitive to gist memory. It was not necessary to remember precisely what the couch looked like, only to remember that there was a couch and to reproduce something that raters would agree looked more like a couch (any couch) than a saw (or a plug, etc.). In contrast, such gist knowledge would be useless during the recognition task. In this case, a figure was scored as correct only if the exact couch was identified among a field of four very similar couches, meaning that success would require detailed visual information. One might speculate that the task-based generation effect might exist for pictures, but be restricted to the kinds of local detailing tapped by our recognition task. In contrast, the "aha" effect may be restricted to holistic, global information.

A final possible theoretical link we propose focuses on the "aha" encoding context and typical generation tasks as problems to be solved. In both cases, there is information that is missing, and hence a degree of ambiguity, and it is up to the subject to attempt a resolution. For example, Jacoby (1978) found that the subjects who were provided the solution (e.g., "table") immediately followed by a word fragment (e.g., "t__ le") demonstrated no greater memory for the generated words than for comparable read items. Jacoby argued that for these massed items, the generation of a word fragment immediately subsequent to reading the solution resulted in a "trivial" processing task. Alternatively, foreknowledge of the word fragments, analogous to the preview manipulation in the present studies, could have suppressed an "aha" effect. Although admittedly speculative, it is reasonable to consider the possibility that encoding mechanisms based on "aha" processes underlie the more typically documented generation effects (see also Soraci et al., 1999). In this sense, "aha" and generation could be regarded as points on a greater problem-solving continuum with "aha" characterized as a solution that requires a considerable reconfiguration of the problem representation and typical generation contexts requiring minimal reconfiguration. Importantly, both involve effort toward comprehension.

\section{REFERENCES}

Auble, P. M., Franks, J. J., \& Soraci, S. A. J. (1979). Effort toward comprehension: Elaboration or "aha!"? Memory \& Cognition, 7, 426434.

BRUNER, J. C., \& POTTER, M. C. ( 1964). Interference in visual recognition. Science, 144, 424-425.

Engel a amp, J., Zimmer, H. D., Mohr, G., \& Sellen, O. (1994). Memory of self-performed tasks: Self-performing during recognition. Memory \& Cognition, 22, 34-39.

Glisky, E. L., \& Rabinowitz, J. C. (1985). Enhancing the generation effect through repetition of operations. Journal of Experimental Psychology: Learning, Memory, \& Cognition, 11, 193-205.

GRAF, P. (1982). The memorial consequences of generation and transformation. Journal of Verbal Learning \& Verbal Behavior, 21, 539548.

$\mathrm{J}_{\mathrm{ACOBY}}$ L. M. (1978). On interpreting the effects of repetition: Solving a problem versus remembering a solution. Journal of Verbal Learning \& Verbal Behavior, 17, 649-667.

JOHNS, E. J., \& SWANSON, L. G. (1988). The generation effect with nonwords. Journal of Experimental Psychology: Learning. Memory, \& Cognition, 14, 180-190.

McDaniel, M. A., Waddil., P. J., \& Einstein, G. O. (1988). A contextual account of the generation effect: A three-factor theory. Journal of Memory \& Language, 27, 521-536.

McEl.ROY, L. A. (1987). The generation effect with homographs: Evidence for postgeneration processing. Memory \& Cognition, 15, 148153.

MCNamara, D. S., \& Healy, A. F. (1995). A procedural explanation of the generation effect: The use of an operand retrieval strategy for multiplication and addition problems. Journal of Memory \& Language, 34, 399-416.

Morris, C. D., Bransford, J. D., \& Franks, J. J. (1977). Levels of pro- 
cessing versus transfer appropriate processing. Journal of Verbal Learning \& Verbal Behavior, 16, 519-533.

PeynircioĞLu, Z. F. (1989). The generation effect with pictures and nonsense figures. Acta Psychologica, 70, 153-160.

Roenker, D. L., Wenger, S. K.. Thompson, C. P., \& Watkins, B (1978). Depth of processing: When the principal of congruity fails Memory \& Cognition, 6, 288-295.

SlameCka, N. J., \& GRAF, P. (1978). The generation effect: Delineation of a phenomenon. Journal of Experimental Psychology: Human Learning \& Memory, 4, 592-604.

Smith, R. W., \& Healy, A. F. (1998). The time-course of the generation effect. Memory \& Cognition, 26, 135-142.
Soraci, S. A., Carlin, M. T., Chechile, R. A., Franks, J. J., Wills, T., \& Watanabe, T. (1999). Encoding variability and cuing in generative processing. Journal of Memory \& Language, 41, 541-559.

Soraci, S. A., Franks, J. J., Bransford, J. D., Chechile, R. A., Belli, R. F., CARR, M., \& CARLIN, M. (1994). Incongruous item generation effects: A multiple-cue perspective. Journal of Experimental Psychology: Learning, Memory, \& Cognition, 20, 67-78.

(Manuscript received September 27, 1998; revision accepted for publication August 16, 1999.) 